\title{
Stable Maintenance of Multiple Plasmids in E. coli Using a Single Selective Marker
}

\author{
Calvin M. Schmidt, ${ }^{\dagger}$ David L. Shis, ${ }^{\dagger}$ Truong D. Nguyen-Huu, ${ }^{\dagger}$ and Matthew R. Bennett ${ }^{*}, \dagger$ \\ ${ }^{\dagger}$ Department of Biochemistry \& Cell Biology, Rice University, Houston, Texas, United States \\ ${ }^{\ddagger}$ Institute of Biosciences \& Bioengineering, Rice University, Houston, Texas, United States
}

ABSTRACT: Plasmid-based genetic systems in Escherichia coli are a staple of synthetic biology. However, the use of plasmids imposes limitations on the size of synthetic gene circuits and the ease with which they can be placed into bacterial hosts. For instance, unique selective markers must be used for each plasmid to ensure their maintenance in the host. These selective markers are most often genes encoding resistance to antibiotics such as ampicillin or kanamycin. However, the simultaneous use of multiple antibiotics to

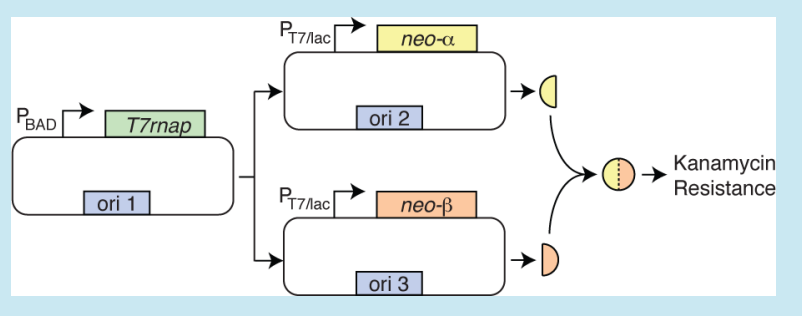
retain different plasmids can place undue stress on the host and increase the cost of growth media. To address this problem, we have developed a method for stably transforming three different plasmids in E. coli using a single antibiotic selective marker. To do this, we first examined two different systems with which two plasmids may be maintained. These systems make use of either T7 RNA polymerase-specific regulation of the resistance gene or split antibiotic resistance enzymes encoded on separate plasmids. Finally, we combined the two methods to create a system with which three plasmids can be transformed and stably maintained using a single selective marker. This work shows that large-scale plasmid-based synthetic gene circuits need not be limited by the use of multiple antibiotic resistance genes.

KEYWORDS: plasmids, antibiotic resistance, kanamycin, T7 RNA Polymerase, split proteins

$\mathrm{T}$ he creation of synthetic gene circuits in Escherichia coli is most often done with the use of plasmids. The ease with which plasmids can be transformed into E. coli and the many tools that are available for manipulating their DNA sequences make them a robust platform for designing, testing and implementing regulatory architectures. Indeed, many previously reported synthetic gene circuits have been constructed on plasmids, including toggle switches, ${ }^{1,13}$ gene oscillators, ${ }^{2,11,21}$ light-sensitive networks ${ }^{17,23}$ and logic gates. ${ }^{24}$ However, as the size and complexity of synthetic gene circuits increase, so too does the need for plasmid-based frameworks that can simultaneously maximize the amount of DNA and minimize the strain on the host.

Plasmid-based synthetic gene circuits frequently use multiple plasmids to house the necessary DNA. In order to stably maintain multiple plasmids within the host, each plasmid type must contain a unique selective marker. For instance, synthetic gene circuits that use two plasmids often contain one plasmid that confers resistance to ampicillin and another plasmid conferring resistance to kanamycin. ${ }^{11,21}$ While these two selective markers work well together, there are some limitations. For instance, long-term cultures of cells containing ampicillin resistance can spawn cheater cells that have lost the plasmid containing $b l a,{ }^{10}$ the gene encoding $\beta$-lactamase. Further, the metabolic burden of producing the proteins necessary to impart resistance to multiple antibiotics can exhaust cellular resources and slow growth. ${ }^{3,4,7}$

Currently, there exist several methods for stably maintaining a single plasmid type without resorting to antibiotics. ${ }^{25}$ Most of these methods involve modifying the chromosome of the host. For instance, one method is to remove an essential gene, such as infA or $g l y A$, from the chromosome and include it on the plasmid. ${ }^{14,26}$ Another method represses the essential gene dapD by placing it under control of the lac promoter. ${ }^{8}$ This repression is then alleviated by transforming in a plasmid containing lacO sites that compete with the promoter for LacI occupancy.

In this study we examined methods for stably maintaining multiple plasmid types using a single selective marker, here the antibiotic kanamycin. We constructed and characterized three different synthetic gene circuits that confer antibiotic resistance to the host if and only if multiple plasmids are present. Each circuit is designed so that no single plasmid can produce resistance in the absence of the others. These systems can easily be used in many different $E$. coli strains, provided those strains contain no source of T7 RNA polymerase (T7RNAP).

The first two designs each require two different plasmids to confer kanamycin resistance. In one of these, we used the RNA polymerase from phage $\mathrm{T} 7$ to drive expression of the gene (neo) encoding aminoglycoside phosphotransferase (3')-IIa (NptII), which confers resistance to kanamycin. By placing the gene encoding T7RNAP on one plasmid, and a T7RNAP specific promoter driving neo on the other, both plasmids are

Special Issue: Engineered Microbes for Therapeutic Applications

Received: June 28, 2012

Published: August 30, 2012 

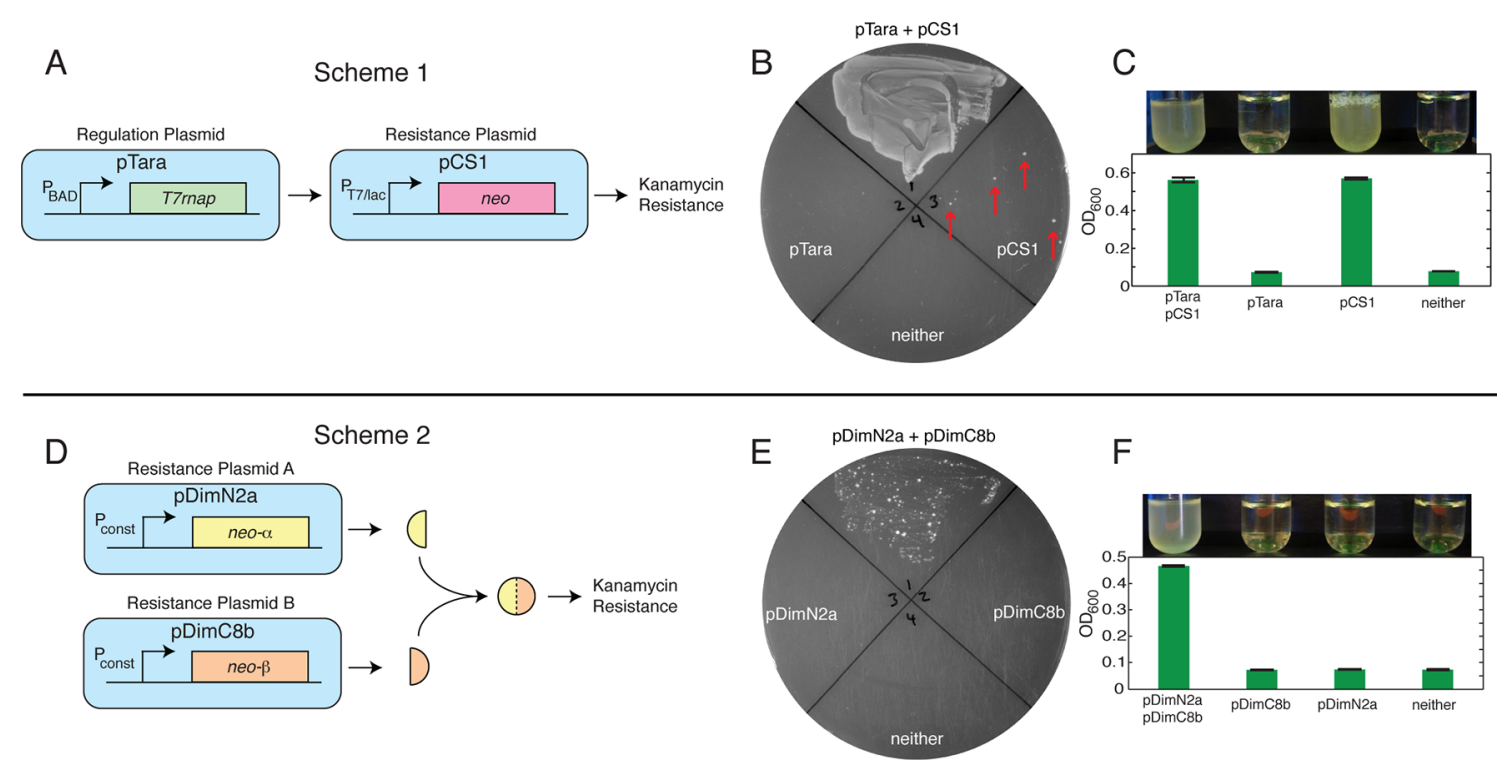

Figure 1. Two circuit designs for stable maintenance of two plasmids. (A) Scheme 1: The first plasmid produces T7RNAP, which is required for the transcription of the neo gene on the second plasmid. (B) Growth on kanamycin plates of cells containing both pTara and pCS1 (top quarter), pTara (left), pCS1 (right) or neither (bottom). While the full system confers resistance, several colonies (red arrows) arise when just pCS1 is present. (C) Liquid culture experiments show the pattern as the plating experiments. Shown is a picture of a representative sample of culture tubes containing cells transformed with the various combinations of the two plasmids (top) and their respective $\mathrm{OD}_{600}$ measurements (below). (D) Scheme 2: The neo gene is split to produce proteins that are enzymatically active only as a dimer. One plasmid contains the $\mathrm{N}$-terminal portion (neo- $\alpha$ ), while the other plasmid contains the C-terminal portion $(n e o-\beta)$. (E) and (F) Plating and liquid culture experiments similar to (B) and (C). Unlike Scheme 1, Scheme 2 shows no mutations that confer kanamycin resistance (see text for details). Photographs in (B), (C), (E) and (F) are representative samples of at least three experiments. Error bars in (C) and (F) are the standard deviations from at least three separate cultures.

required for kanamycin resistance. In the other two-plasmid network, we examined a previously reported split NptII system, with the genes encoding either half of the enzyme being placed on two different plasmids. ${ }^{19}$ Finally, we constructed a circuit that combines the two above systems to create a system that requires three different plasmids. To do this, we replaced the constitutive promoters driving the two halves of the split neo gene above with promoters targeted by T7RNAP. Therefore, in addition to the plasmids containing the two halves of neo, a third plasmid encoding T7RNAP is required to confer kanamycin resistance.

We find that stable maintenance of multiple plasmids using a single selective marker (in this case kanamycin) is possible. Each of the three systems is not only able to confer kanamycin resistance to the host, but also capable of being maintained during long-term serial dilution experiments. In addition, we find these systems can be directly transformed into their host and plated onto media containing only kanamycin. This capability alleviates the need for "back-up" resistance markers on each plasmid and thereby reduces the necessary size and metabolic load of each plasmid.

We first designed a scheme (Scheme 1, Figure 1A) in which one plasmid, pTara, houses the gene encoding T7RNAP driven by the arabinose-inducible $\mathrm{P}_{\mathrm{BAD}}$ promoter. ${ }^{27}$ In addition, a second plasmid, pCS1, contains the neo gene driven by a hybrid $\mathrm{P}_{\mathrm{T} 7 / \text { lac }}$ promoter. Since $\mathrm{T} 7$ promoters are orthogonal to native E. coli RNA polymerases, ${ }^{22}$ transcription of the neo gene is initiated only in the presence of T7RNAP. In addition, the $\mathrm{P}_{\mathrm{T} 7 / \mathrm{lac}}$ promoter contains a LacI operator site to allow control over the amount of transcription through titration of isopropyl $\beta$-D-1-thiogalactopyranoside (IPTG). ${ }^{9}$ Because transcription of neo requires both plasmids, host strains will be resistant to kanamycin only if both plasmids are present.
We found that Scheme 1 could confer resistance to kanamycin. To test this, cells were transformed with either the full system (pTara and pCS1) or a partial system (pTara or pCS1 or neither). The bacteria were streaked onto solid lysongeny broth (LB) agar plates containing kanamycin and passed into liquid LB with kanamycin. Cultures were then allowed to grow overnight. When plated onto media with kanamycin, as shown in Figure 1B, the full system shows robust growth. However, we also found that it is susceptible to mutation. While cells transformed with only pTara or neither plasmid showed no growth, several colonies were observed with cells transformed with just pCS1. Similar results were observed when these strains were grown in liquid cultures (Figure 1C).

Upon sequencing, it was found that cells transformed with just $\mathrm{pCS} 1$ and grown in the presence of kanamycin had obtained mutations to the T7 promoter driving neo. Specifically, we found that an IS10 insertion sequence had been placed into the promoter. Upon induction of cellular stress, the IS10 sequence is randomly inserted into DNA by Tn10 transposase. ${ }^{12}$ It is not site specific, as sequencing showed that the insertion sequence was not always inserted into the same position on the plasmid. It was usually inserted into a position near the beginning of the $\mathrm{T} 7$ promoter, presumably disrupting the orthogonality of the $\mathrm{T} 7$ promoter and allowing native E. coli RNAP to transcribe neo. No similar mutations were ever noticed when both pTara and pCS1 were transformed together and relied upon to confer kanamycin resistance, even after 30 days of serial dilution (see below).

The design for Scheme 2 (Figure 1D) utilizes a split version of neo. ${ }^{19}$ This split version of neo has an N-terminal fragment encoding residues $1-59$ (neo- $\alpha$ ) of NptII and a C-terminal fragment encoding residues 59-264 (neo- $\beta$ ). In addition, both halves contain a leucine zipper fusion to promote dimerization 


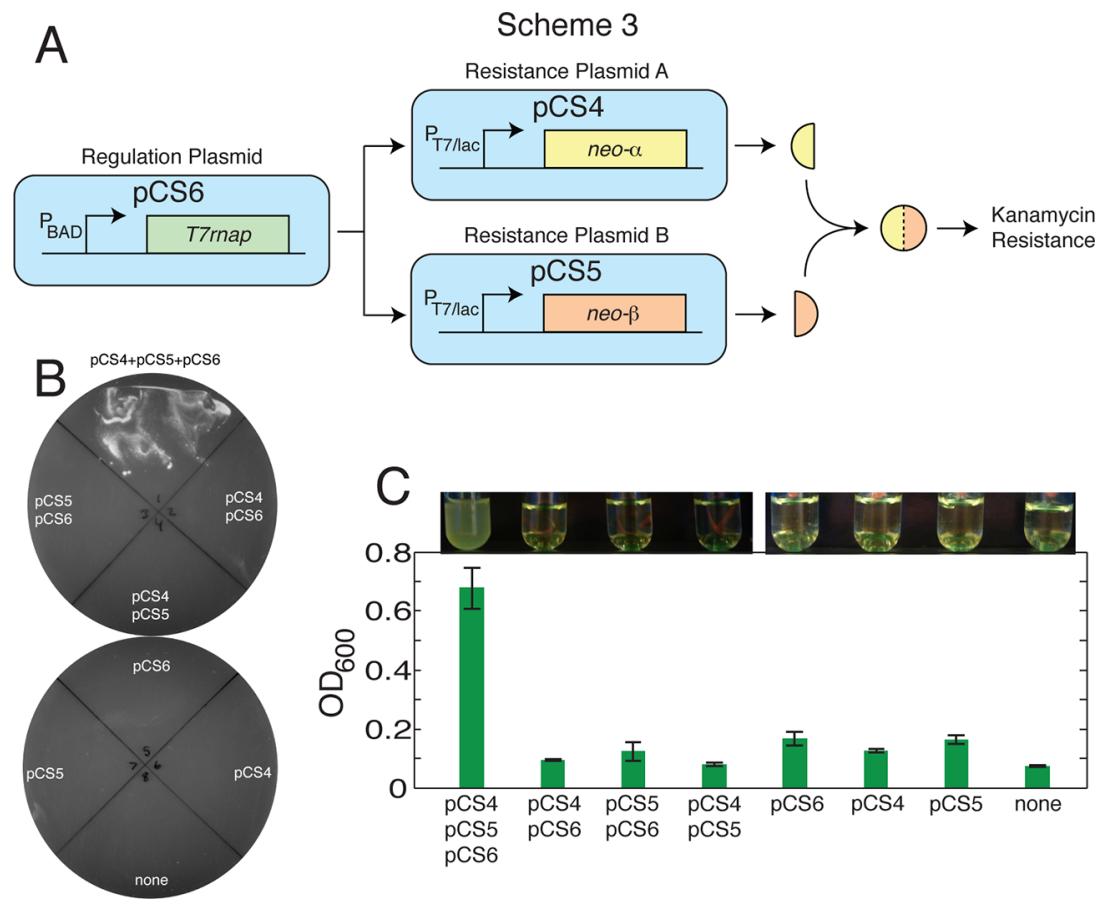

Figure 2. The three-plasmid system. (A) Scheme 3: Circuit design for stable maintenance of three plasmids. The two halves of the Neo gene from scheme three are placed under the control of the $\mathrm{P}_{\mathrm{T} 7 / \text { lac }}$ promoter. Therefore, the plasmid containing T7RNAP from Scheme 1 is also required for resistance to kanamycin. (B) Cells streaked onto plates containing only kanamycin shows that only those cells containing all three plasmids are capable of growing. (C) Cells grown in liquid LB with kanamycin only proliferate when all three plasmids are present. No growth was ever seen in either liquid or on plates when one, two or all three plasmids were absent. Photographs in (B) and (C) are representative samples of at least three experiments. Error bars in (C) are the standard deviations from three separate cultures.

of the two fragments. Neither half of the split NptII is enzymatically active, but the dimer confers resistance to kanamycin. Therefore, host strains will survive in the presence of kanamycin only if both plasmids are present.

Growth experiments with Scheme 2 showed that both plasmids were required for growth in the presence of kanamycin, both on plates (Figure 1E) and in liquid (Figure $1 \mathrm{~F}$ ). In addition, no mutations were ever observed allowing either of the two plasmids, in the absence of the other, to confer kanamycin resistance to the host.

We next designed a system that requires three plasmids to confer kanamycin resistance. Scheme 3, shown in Figure 2A, uses a combination of Schemes 1 and 2 . By replacing the constitutive promoters that drive expression of neo- $\alpha$ and neo- $\beta$ from Scheme 2 with the $\mathrm{P}_{\mathrm{T} 7 / \text { lac }}$ promoter, the system now requires T7RNAP to be supplied by a third plasmid, as in Scheme 1 .

As with the two-plasmid systems, we tested whether or not our three-plasmid circuit was necessary and sufficient for robust growth in the presence of kanamycin. We examined the growth of various strains containing all eight possible combinations of the three plasmids. When grown on plates (Figure $2 \mathrm{~B}$ ) or in liquid media (Figure 2C), we found that only those cells containing the full system grew.

Interestingly, unlike Scheme 1, which also relies on T7RNAP, the three-plasmid system did not show susceptibility to mutation while lacking one or more of the plasmids. In particular, since cells lacking T7RNAP in the two-plasmid system were able to mutate and grow in kanamycin, we were concerned that the three-plasmid system had a similar vulnerability. When cells transformed with the two plasmids containing neo- $\alpha$ and neo- $\beta$ driven by the T7 promoter (pCS4 and pCS5) were grown in the presence of kanamycin, no mutations were ever observed that conferred resistance. This is perhaps due to the split nature of the resistance gene and the fact that transposon insertion is a rare event. In other words, while the neo containing plasmid from Scheme 1 (pCS1) only requires a single insertion event, $\mathrm{pCS} 4$ and pCS5 require two such events to occur nearly simultaneously to confer resistance in the absence of T7RNAP.

Each plasmid of the three schemes described above contained a "backup" antibiotic marker. These backup resistance genes aided in cloning each plasmid and allowed us to first transform them into strains before testing the efficacy of kanamycin resistance. However, we wanted to test whether or not these backup resistance markers were essential and if the schemes could be plated directly onto kanamycin plates after transformation.

We found that each system could be transformed into competent cells and plated directly onto kanamycin plates. However, the transformation efficiency of each system was significantly less when plated onto kanamycin than when plated onto the back-up antibiotics, as shown in Figure 3. For example, the two plasmids comprising Scheme 1 contained back-up markers conferring resistance to ampicillin and chloramphenicol. After cotransforming pTara and pCS1 into competent cells, the outgrowth was split and plated either onto LB agar with ampicillin and chloramphenicol or onto LB agar with just kanamycin. When compared to plating onto the back-up resistance markers, the transformation efficiency of Scheme 1 was just $13 \%$ (Figure 3A).

Similar results were obtained when examining Schemes 2 and 3 , as shown in Figure $3 \mathrm{~B}$ and $\mathrm{C}$, respectively. The relative transformation efficiency of Scheme 2 was just 3\%. For Scheme 

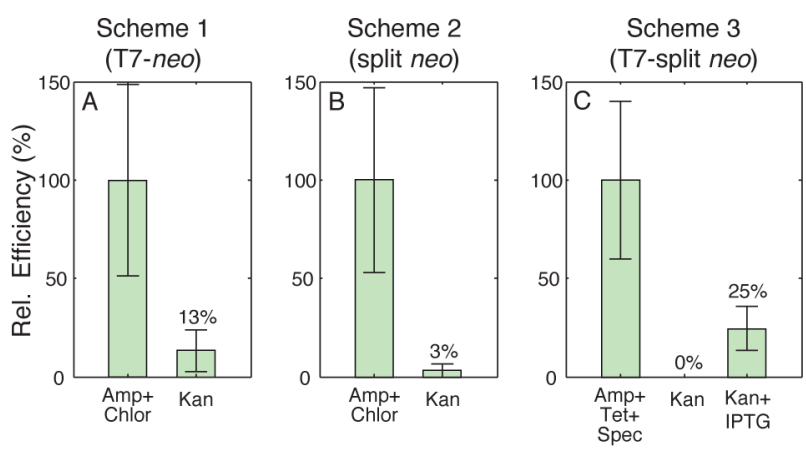

Figure 3. Transformation efficiency of the multiplasmid schemes. (A) Relative transformation efficiency of Scheme 1 . When cells were plated directly onto kanamycin plates (right), the efficiency was roughly $13 \%$ of the efficiency when plated onto dual-selection plates (left). The dual-selection plate contained ampicillin and chloramphenicol, the back-up resistance markers for the two plasmids in Scheme 1. (B) The transformation efficiency of Scheme 2 when plated onto kanamycin plates (right) was $3 \%$ of that when plated onto dual selection plates (left). (C) Relative transformation efficiency of Scheme 3. Here, we found no colonies when plated directly onto kanamycin plates. However, when $1 \mathrm{mM}$ IPTG (which induces the $\mathrm{P}_{\mathrm{T} 7 / \mathrm{lac}}$ promoter) was added to the kanamycin plates, the relative efficiency was $25 \%$.

3 , the three back-up resistance genes conferred resistance to ampicillin, tetracycline and spectinomycin. Co-transformation of the three plasmids in Scheme 3 onto plates containing all 3 back-up markers was possible, but we obtained no colonies when we plated onto kanamycin only. However, when we added $1 \mathrm{mM}$ IPTG, we found robust growth, with a relative transformation efficiency of approximately $25 \%$. Note that while IPTG, which helps to induce the $\mathrm{P}_{\mathrm{T} 7 / \mathrm{lac}}$ promoter, is necessary to transform Scheme 3 directly onto kanamycin, it is not necessary for growth in liquid culture. In fact, cells containing Scheme 3 grown in liquid culture with kanamycin grow decidedly better in the absence of IPTG, as shown in Figure 4. Similar results were obtained with Scheme 1 in liquid culture.

Though the rates for transforming these circuits directly onto kanamycin was significantly lower than onto their back-up markers, it should be noted that when concentrations of plasmids are similar to that which can be obtained with a

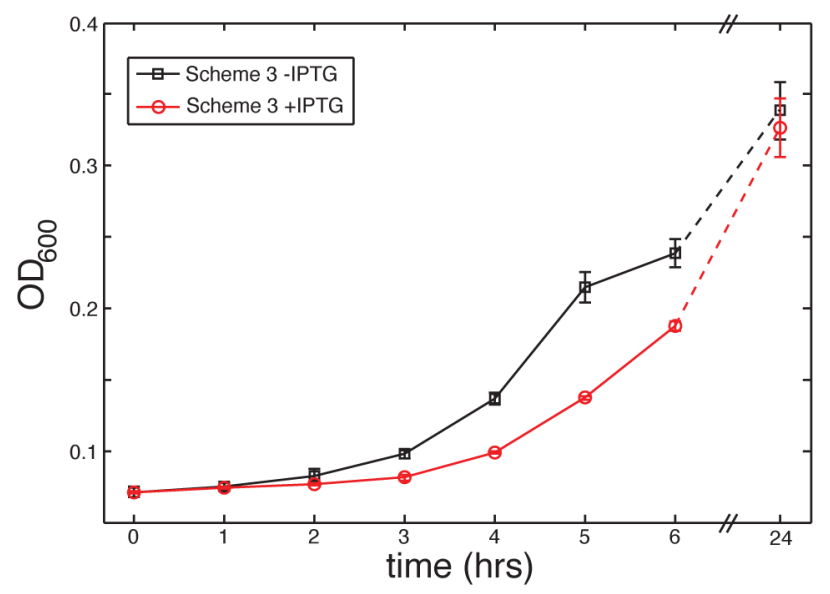

Figure 4. Growth of Scheme 3 in liquid culture. The growth of Scheme 3 in liquid culture containing kanamycin is significantly better in the absence of IPTG (black lines, squares) than in the presence of 1 mM IPTG (red line, circles). standard purification method, the number of colonies of DH10B electrocompetent transformants on kanamycin is so great that it is nearly impossible to distinguish single colonies. This indicates that removal of the individual resistance genes prior to final assembly would be possible.

As the growth rate was affected by the levels of transcription by T7 RNAP (as induced by IPTG), we hypothesized that putting T7RNAP on a plasmid with an origin of replication that confers a lower copy might result in increased cell growth. pTara contains a p15a origin of replication, which confers a copy number of approximately 15 per cell. ${ }^{16}$ We therefore placed the promoter and gene driving expression of T7RNAP into a vector that has a pSC101 origin, which confers a copy number of approximately 5 per cell. ${ }^{15}$ This new scheme we called Scheme 1LC (Low Copy). We then measured the growth rates of cells containing either Scheme 1 or Scheme $1 \mathrm{LC}$ in the presence or absence of $1 \mathrm{mM}$ IPTG, as shown in Figure 5.

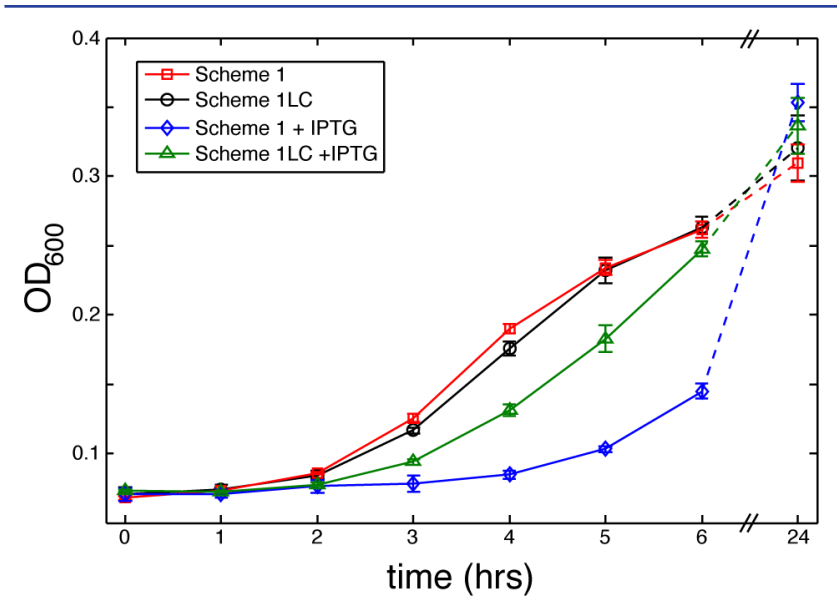

Figure 5. The effect of copy number on growth. The growth of Scheme 1 and Scheme 1LC are nearly identical in the absence of IPTG (red and black curves, respectively). The presence of $1 \mathrm{mM}$ IPTG drastically reduces the growth of cells containing Scheme 1 (blue curve). Scheme 1LC does better in the presence of IPTG (green curve) but still worse than either in the absence of IPTG.

For uninduced cells, there was no difference in growth rate (Figure 5 red and black curves). However, Scheme 1LC significantly outgrew Scheme 1 when we induced the cells with $1 \mathrm{mM}$ IPTG (Figure 5 green and blue curves). Therefore, while some increase in growth rate is attainable when T7RNAP is expressed off of a low copy number in the presence of IPTG, growth was best in its absence.

We next wanted to test whether or not each scheme could be used to stably maintain the plasmids within the hosts during long-term serial dilution experiments. To do this, we performed serial dilution experiments on each scheme in liquid LB containing only kanamycin (see Methods section). Each morning the overnight cultures were used to seed the next round, and then plasmid DNA was extracted, digested and run through an agarose gel to determine the presence or absence of each plasmid. The results of these experiments are shown in Figure 6.

We found that each scheme could be stably maintained with only kanamycin during serial dilution for many weeks. For instance, Scheme 1 maintained both pTara and pCS1 for more than a month (Figure 6A, left and right). This maintenance was 


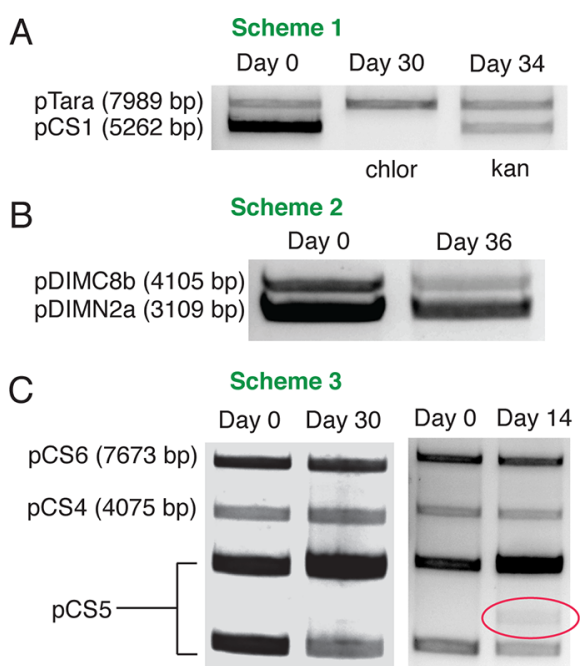

Figure 6. Long-term stability during serial dilution experiment. (A) Image of an agarose gel containing digested plasmid DNA from one serial dilution experiment. pTara and pCS1 were both stably maintained in the presence of only kanamycin. The bands that were present at day 0 (left) were still present at day 34 after continuous selection with kanamycin (kan). When chloramphenicol (chlor) was used to select only for pTara and not for pCS1, pCS1 was lost. (B) Scheme 2 was maintained in the presence of kanamycin for at least 36 days. (C) Two separate serial dilution experiments with Scheme 3. In the experiment on the left, Scheme 3 was maintained in the presence of kanamycin for 30 days. In the right experiment, the plasmids comprising Scheme 3 are still present in the culture, but an unknown faint band appeared (red circle) after 2 weeks.

not just a remnant of nonselected plasmid maintenance, as cultures grown in the presence of only chloramphenicol (which selects for pTara and not pCS1) eventually lose pCS1 (Figure $6 \mathrm{~A}$, middle). In the split NptII system, Scheme 2, both plasmids persisted during serial dilution in the presence of only kanamycin for well over a month, as shown in Figure 6B. For both Schemes 1 and 2, six serial dilution experiments yielded identical results.

The maintenance of Scheme 3 also lasted for at least 14 days, and sometimes for at least 30 days, in the presence of kanamycin (Figure 6C). However, in some trials we saw the emergence of faint bands in the agarose gels (Figure 6C, red circle), usually around day 14 . We were unable to sufficiently identify these bands and therefore do not know what they are. However, it should be noted that the new bands remained faint and the bands corresponding to the three plasmids that make up Scheme 3 always persisted for at least 30 days throughout each of the six serial dilution experiments performed.

There are many reasons to avoid using multiple antibiotics when implementing large-scale synthetic gene circuits in bacteria, from reducing stress and metabolic load on cells to decreasing the cost of growth media. Here, we showed that stable maintenance of multiple plasmids need not require multiple antibiotics. The systems we examined are simple to use, in that they can be directly transformed into any competent cell strain that lacks kanamycin resistance. This means that the removal of backup resistance markers is possible after the subcloning of each plasmid in the system is complete. Furthermore, the long-term growth of cells in liquid culture is also possible, as the Schemes we examined maintained their plasmids in the presence of kanamycin for many weeks. In fact, we never saw cultures drop any plasmid in either of the twoplasmid Schemes.

Perhaps the real utility of these Schemes is their possible use in creating antibiotic-free systems for multiple plasmid maintenance. This might be done by combining one of the Schemes examined above with one of the currently existing methods that are used to generate antibiotic-free maintenance of single plasmids. For instance, if the neo gene in Scheme 1 were replaced with an essential chromosomal gene, then the two plasmids would be required for cellular growth in an appropriate knockout strain. Similar schemes for maintaining three plasmids without antibiotics are also conceivable.

\section{METHODS}

Plasmid Construction. pTara was obtained from Dr. Kathleen Matthews. ${ }^{27}$ pDimN2a and pDimC8b were obtained from Dr. Marc Ostermeier and originally named pDIM-N2Neo[1-59]N58S and pDIM-C8-Neo[59-264]R177S,V198E, respectively. ${ }^{19}$ We used shortened names for brevity. pCS1 was made by PCR amplifying the neo gene from a pET28 vector and then ligating it back into pET28 using the sites NcoI and BamHI to place it under control of the T7 promoter. The resulting T7-Neo cassette was then PCR amplified and ligated into $\mathrm{pBR} 322^{5}$ in the place of the Tet resistance gene using circular polymerase extension cloning (CPEC). ${ }^{20} \mathrm{pCS} 4$ was made by PCR amplifying the neo- $\alpha$ gene from pDIMN 2 and ligating into pET28 using the sites $\mathrm{NcoI}$ and BamHI. The resulting T7- neo- $\alpha$ cassette, along with lacI, was then PCR amplified and added into pACYC177 ${ }^{6}$ in place of the kanamycin resistance gene using CPEC. pCS5 was made by PCR amplifying the neo- $\beta$ gene from pDIMN2 and ligating into pET28 using the sites NcoI and BamHI. The T7- neo- $\beta$ cassette was then PCR amplified and ligated into pBR322 in the place of the bla gene using the sites AseI and EcoRI. pCS6 was made by PCR amplifying the genes encoding T7RNAP and AraC from $\mathrm{pTara}$ and inserting the resulting fragment into $\mathrm{pZS} 4{ }^{18}$ in the place of lacI and tetR using CPEC.

Plasmid and Cell Maintenance. Cells were grown in standard liquid LB or on LB agar plates. The antibiotics were used at concentrations of $100 \mu \mathrm{g} / \mathrm{mL}$ for ampicillin, $50 \mu \mathrm{g} / \mathrm{mL}$ for kanamycin, $50 \mu \mathrm{g} / \mathrm{mL}$ for chloramphenicol, $50 \mu \mathrm{g} / \mathrm{mL}$ for spectinomycin, and $20 \mu \mathrm{g} / \mathrm{mL}$ for tetracycline. IPTG was used at a concentration of $1 \mathrm{mM}$. Note that while the gene encoding T7RNAP in Schemes 1 and 3 is driven by the arabinoseinducible $\mathrm{P}_{\mathrm{BAD}}$ promoter, we found that the basal expression level in the absence of arabinose produced enough T7RNAP for the circuits to function.

Testing Cell Growth in Culture. Five milliliters of liquid LB cultures containing appropriate antibiotics and inducers were inoculated with $50 \mu \mathrm{L}$ of DH10B cells grown overnight in LB. These cells had been previously transformed with different plasmids. Growth was measured by transferring $100 \mu \mathrm{L}$ of culture to a 96 well plate and taking $\mathrm{OD}_{600}$ measurements using a TECAN Infinite M1000 plate reader. Measurements were taken every hour for $6 \mathrm{~h}$, and again at $24 \mathrm{~h}$ after inoculation. Cultures were grown in a $37{ }^{\circ} \mathrm{C}$ incubator with shaking.

Testing Circuit Transformation Efficiency. DH10B electrocompetent cells were transformed with $1 \mu \mathrm{L}$ of DNA containing plasmids comprising the three different gene circuits. The outgrowth of these cells $(\approx 400 \mu \mathrm{L})$ was split, with $200 \mu \mathrm{L}$ plated on LB agar plates containing kanamycin, and $200 \mu \mathrm{L}$ plated on plates containing antibiotics that select separately for each plasmid in the circuit. For the circuit 
selecting for three plasmids, the split outgrowth was plated on kanamycin and kanamycin +1 mM IPTG plates, while $200 \mu \mathrm{L}$ from another transformation was plated on spectinomycin + tetracycline + ampicillin plates. Cells were grown overnight at $37{ }^{\circ} \mathrm{C}$ before counting colonies.

Testing Viability of Individual Plasmids. Every plasmid combination, including single plasmids of the dual plasmid systems, and combinations of two plasmids of the three plasmid system were transformed into $\mathrm{DH} 10 \mathrm{~B}$ cells and plated on $\mathrm{LB}$ agar plates containing antibiotics selecting for the individual plasmids. Colonies from these plates were picked into liquid LB containing the same antibiotics and grown overnight. These cultures were passed into liquid LB with kanamycin and streaked on LB agar plates with kanamycin.

Serial Dilution Experiments. Plasmids were transformed into DH10B cells and plated on LB agar plates selecting for the individual antibiotic markers for each plasmid. Single colonies were picked into $3 \mathrm{~mL}$ of liquid LB cultures containing individually selecting antibiotics and grown overnight in a shaking incubator at $37^{\circ} \mathrm{C}$ and $200 \mathrm{rpm}$. A 1:600 dilution was created by passing $5 \mu \mathrm{L}$ of the overnight culture into $3 \mathrm{~mL}$ of liquid LB with kanamycin. They were passed into new media in this manner every $24 \mathrm{~h}$ for the duration of the experiments.

\section{AUTHOR INFORMATION}

\section{Corresponding Author}

*E-mail: matthew.bennett@rice.edu.

\section{Notes}

The authors declare no competing financial interest.

\section{ACKNOWLEDGMENTS}

We thank Dr. Kathleen Matthews for use of pTara and Dr. Marc Ostermeier for the split neo plasmids. We also thank Dr. Joff Silberg for illuminating discussions regarding this work. Funding was provided by the Welch Foundation, grant number C-1729.

\section{REFERENCES}

(1) Atkinson, M. R., Savageau, M. A., Myers, J. T., and Ninfa, A. J. (2003) Development of genetic circuitry exhibiting toggle switch or oscillatory behavior in Escherichia coli. Cell 113, 597-607.

(2) Balagaddé, F. K., You, L., Hansen, C. L., Arnold, F. H., and Quake, S. R. (2005) Long-term monitoring of bacteria undergoing programmed population control in a microchemostat. Science 309, 137-140.

(3) Bentley, W. E., Mirjalili, N., Anderson, D. C., Davis, R. H., and Kompala, D. S. (1990) Plasmid-encoded protein: the principal factor in the 'metabolic burden' associated with recombinant bacteria. Biotechnol. Bioeng. 35, 668-681.

(4) Birnbaum, S., and Bailey, J. E. (1991) Plasmid presence changes the relative levels of many host cell proteins and ribosome components in recombinant Escherichia coli. Biotechnol. Bioeng. 37, 736-745.

(5) Bolivar, F., Rodriguez, R. L., Greene, P. J., Betlach, M. C., Heyneker, H. L., Boyer, H. W., Crosa, J. H., and Falkow, S. (1977) Construction and characterization of new cloning vehicles. II. A multipurpose cloning system. Gene 2, 95-113.

(6) Chang, A. C., and Cohen, S. N. (1978) Construction and characterization of amplifiable multicopy DNA cloning vehicles derived from the P15A cryptic miniplasmid. J. Bacteriol. 134 (3), $1141-1156$

(7) Corchero, J. L., and Villaverde, A. (1998) Plasmid maintenance in Escherichia coli recombinant cultures is dramatically, steadily, and specifically influenced by features of the encoded proteins. Biotechnol. Bioeng. 58, 625-632.
(8) Cranenburgh, R. M., Hanak, J. A. J., Williams, S. G., and Sherratt, D. J. (2001) Escherichia coli strains that allow antibiotic-free plasmid selection and maintenance by repressor titration. Nucleic Acids Res. 29, e26.

(9) Dubendorf, J. W., and Studier, F. W. (1991) Controlling basal expression in an inducible $\mathrm{T} 7$ expression system by blocking the target T7 promoter with lac repressor. J. Mol. Biol. 219, 45-59.

(10) Dugatikin, L. A., Perlin, M., Lucas, J. S., and Atlas, R. (2005) Group-beneficial traits, frequency-dependent selection and genotypic diversity: an antibiotic paradigm. Proc. R. Soc. B 272, 79-83.

(11) Elowitz, M. B., and Leibler, S. (2000) A synthetic oscillatory network of transcriptional regulators. Nature 403, 335-338.

(12) Foster, T. J., Davis, M. A., Roberts, D. E., Takeshita, K., and Kleckner, N. (1981) Genetic organization of transposon Tn10. Cell 23, 201-213.

(13) Gardner, T. S., Cantor, C. R., and Collins, J. J. (2000) Construction of a genetic toggle switch in Escherichia coli. Nature 403, 339-342.

(14) Hägg, P., de Pohl, J. W., Abdulkarim, F., and Isaksson, L. A. (2004) A host/plasmid system that is not dependent on antibiotics and antibiotic resistance genes for stable plasmid maintenance in Escherichia coli. J. Biotechnol. 111, 17-30.

(15) Hasunuma, K., and Sekiguchi, M. (1977) Replication of plasmid pSC101 in Eschericia coli K12: Requirement for dnaA function. Mol. Gen. Genet. 154, 225-230.

(16) Hiszczyńska-Sawicka, E., and Kur, J. (1997) Effect of Escherichia coli mutations on plasmid p15A copy number. Plasmid 38, 174-179.

(17) Levskaya, A., Chevalier, A. A., Tabor, J. J., Simpson, Z. B., Lavery, L. A., Levy, M., Davidson, E. A., Scouras, A., Ellington, A. D., Marcotte, E. M., and Voigt, C. A. (2005) Engineering Escherichia coli to see light. Nature 438, 441-442.

(18) Lutz, R, and Bujard, H. (1997) Independent and tight regulation of transcriptional units in Escherichia coli via the LacR/O, the TetR/O and AraC/ $/ \mathrm{I}_{1}-\mathrm{I}_{2}$ regulatory elements. Nucleic Acids Res. 25, 1203-1210.

(19) Paschon, D. E., Patel, Z. S., and Ostermeier, M. (2005) Enhanced catalytic efficiency of amino glycoside phosphotransferase $\left(3^{\prime}\right)$-IIa achieved through protein fragmentation and reassembly. J. Mol. Biol. 353, 26-37.

(20) Quan, J., and Tian, J. (2009) Circular polymerase extension cloning of complex gene libraries and pathways. PLoS One 4, e6441.

(21) Stricker, J., Cookson, S., Bennett, M. R., Mather, W. H., Tsimring, L. S., and Hasty, J. (2008) A fast, robust and tunable synthetic gene oscillator. Nature 456, 516-519.

(22) Studier, F. W., and Moffatt, B. A. (1986) Use of bacteriophage T7 RNA polymerase to direct selective high-level expression of cloned genes. J. Mol. Biol. 189, 113-130.

(23) Tabor, J. J., Salis, H. M., Simpson, Z. B., Chevalier, A. A., Levskaya, A., Marcotte, E. M., Voigt, C. A., and Ellington, A. D. (2009) A synthetic genetic edge detection program. Cell 137, 1272-1281.

(24) Tamsir, A., Tabor, J. J., and Voigt, C. A. (2011) Robust multicellular computing using genetically encoded NOR gates and chemical 'wires'. Nature 469, 212-215.

(25) Vandermeulen, G., Marie, C., Scherman, D., and Préat, V. (2011) New generation of plasmid backbones devoid of antibiotic resistance marker for gene therapy trials. Mol. Ther. 19, 1942-1949.

(26) Vidal, L., Pinsach, J., Striedner, G., Caminal, G., and Ferrer, P. (2008) Development of an antibiotic-free plasmid selection system based on glycine auxotrophy for recombinant protein overproduction in Escherichia coli. J. Biotechnol. 134, 127-136.

(27) Wycuff, D. R., and Matthews, K. S. (2000) Generation of an AraC-araBAD promoter-regulated $\mathrm{T} 7$ expression system. Anal. Biochem. 277, 67-73. 